\title{
Reverse Engineering of Pilot Wheel Vane
}

\author{
Yi-gang $\mathrm{Hu}$ \\ Engineering Training Center \\ Shanghai University of Engineering Science \\ Shanghai, China, 86-21-67791272 \\ 13671595326@126.com
}

\author{
Song Fang \\ Engineering Training Center \\ Shanghai University of Engineering Science \\ Shanghai, China, 86-21-67791247 \\ songfang2005@163.com
}

\begin{abstract}
The pilot wheel vane is one of important parts of hydraulic transmission. Its construction, processing precision and assembly can influence the function of the transmission directly. Since the shape of pilot wheel vane is very complicated, its modeling, processing and measure are the difficult points of engineering aspect. In this paper, the vane points were measured to become the point clouds using three coordinates measurer. Then, according to the procedure of point, curve, face and solid body, the curve face imitation of the vane was made using CAD software. And the 3D modeling of pilot wheel was made.
\end{abstract}

Keywords-vane; reverse engineering; pilot wheel; surface reconstruction

\section{INTRODUCTION}

Reverse Engineering (RE) is the method of engineering design, which can obtain re-structuring 3D model of the part using CAD system from the part body or the greasy filth model to producing the original numerical model through data collecting [1].

The pilot wheel vane is one of important parts of hydraulic transmission. Its construction, processing precision and assembly can influence the function of the transmission directly. Since the shape of pilot wheel vane is very complicated, its modeling, processing and measure are the difficult points of engineering aspect. In this paper, the vane points were measured to become the point clouds using three coordinates measurer. Then, according to the procedure of point, curve, face and solid body, the curve face imitation of the vane was made using UG software. And the 3D modeling of pilot wheel was made using Solid Edge software.

\section{DATA COLLECTING OF VANE}

Data collecting is the base of Reverse Engineering. The method of data collecting: The one is no-attachable, such as triangle measure, using laser emits a light to a point of the face measured, according to the information of reflecting light computes the coordinate of reflecting point. Its character is that can measure easy buckling faces, precision up to $0.01 \mathrm{~mm}$. The another is attachable, such as three coordinates measure(CMM), precision up to $0.005 \mathrm{~mm}$.

As the vane of pilot wheel was measured, the establishing of measure coordinates should consider measure convenient, should decrease measure died areas as far as possible, and should consider the geometry feature of solid body modeling convenient. For CMM, the error of data collecting is mainly itself measure error of CMM, the other errors are the size error produced by the original error of measure coordinate and the error begun by mathematics computation of measure software. To reduce data tidying up work and to make curve face imitation convenient, before measure we draw the lines on the vane face, and then let the measure head measure along these lines, hence the measure points appear with the rule arrangement. If the died area of measure appears, the measure head can not measure to produce data blanks and dada pieces, in this time the multi-view dada makeup can be considered[2].

\section{A. Determining the measure base of pilot wheel}

First, the pilot wheel is located on the platform of three coordinate's measurer. Since the curvature is bigger, to make $\mathrm{X} 、 \mathrm{Y} 、 \mathrm{Z}$ coordinates of inter and outer curve faces measure convenient, the spindle line of the pilot wheel must be vertical to the platform of three coordinates measurer. Three measure bases: $\mathrm{Z}$ coordinate is in line with the spindle line of the pilot wheel, the original locates in the assembly plane of the pilot wheel and the pump wheel, this plane is XY plane. If we only measure the dada of vane, the coordinates may be set at any space post. But besides the vane, there are other structures on the pilot wheel, such as inter and outer curve faces, outline, etc. Hence XY plane is located in the assembly plane of the pilot wheel and the pump wheel, so that the coordinate locations of the other structures of pilot wheel are determined.

\section{B. Measure of pilot wheel vane}

Along the cross line of the vane work face and the vane back face with the flow road, several points (more than ten points) were distributed. The beginning point is set at the cross point, then the measure head begins to measure using three coordinates measurer.

Since the entrance and the exit of the vane are variable radius circular-angle, along the ends of upper and lower cross lines, several points are set again, and the measure is continued. However the curve face coordinates of vane outline are measured. The coordinates of vane outline are very important, and this is the base work to structure the vane outline. Then several coordinates of the vane work face and the vane back face, and several coordinates of the entrance and exit of the vane variable radius circular-angle are obtained. These coordinates are used to adjust the vane curve face and to test the error between the vane curve face structured and the real 
vane curve face. The point cloud is measured, follow as Figure 1.

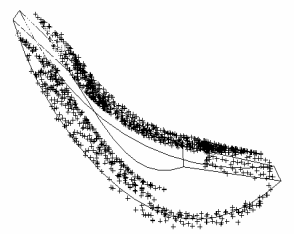

Figure1. The point cloud of vane

\section{CURVE FACE IMITATION OF THE VANE}

Curve face imitation is the key of reverse engineering. The computation of curve face imitation is divided into two classes; the one is function curve face imitation, such as polynomial insert, the precision of this method is high, and this method can be sure to be differential continually for the curve, hence the curve is very sliding. But as there are more insert points, the curve is high rank function; more swings will appear, so that the local mending ability is not good. Another is rectangle region parameter curve face imitation, such as COONS, the curve face is structured with four seal edge curve lines; the insert precision of this method is high. But the mutual vector of insert point will appear, so that the local mending ability is not good. The imitation curve face of three powers $B$ pattern function determined by four control points can make sure of the curve face two ranks to continue. $\mathrm{N}$ control points will produce N-3 curves, and these curves can be mended locally. To provide united mathematics description for analysis curve face and free curve face, the power factor is introduced to increase the local mending ability, NURBS curve face is produced on the base of $\mathrm{B}$ pattern function, and these contents become the standard of CAD/CAM software modeling [3]. As satisfying the curve face precision, the determination of good curve face is the more sliding the better. The slide means the curvature changes uniformly, and two-power derivative is continual. The slide curve face must require the sliding curve. Energy method, local re-spring method and curvature method are three methods of sliding curve [4].

Usually a complicate curve face is imitated through many curve faces; sometimes the edge of curve face is produced through crossing and extending of curve face or curve line. Using CAD software editing function, such as crossing, extending, transiting, cutting out etc. can make sure of no gap between the planes or no piling up each other, and is continual for GC0, GC1, and GC2. And the curve face is required sliding as making sure of the precision.

\section{A. Reading measure data}

The data measured by three coordinate's measurer are conserved with IGES. The first step of curve face reconstruction is to gain the data points of measure. At UG File menu, select "Import", read the data of IGES, show the data cloud in drawing area.

\section{B. Structuring outline of vane}

It is very important to structure pattern curve in curve design. The vane outline is considered as pattern curve. First according to the dada points of vane outline measured by three coordinate's measurer, the pattern curve is produced using UG Toolbox/spline/Through Points edicts, and the rank of the pattern curve is 3-4. Then the curve face is produced by the pattern curve, and the pattern curve is very important to be considered as intermediate process. The points of producing the pattern are made as the control points, and each control point determines the direction of the pattern curve. Since there is the cast circular-angle at the cross line of the vane, it will influence the measure precision, and hence the pattern curve is dealt with sliding, and let the pattern curve continue in two-rank derivative. Modeling tolerance contains distance tolerance and angle tolerance. Some references suggest the tolerance be set in $0.01 \mathrm{~mm}-0.001 \mathrm{~mm}$; it is suitable to make sure of the precision modeling [5]. The shape of the vane outline is drawn, follow as Figure 2.

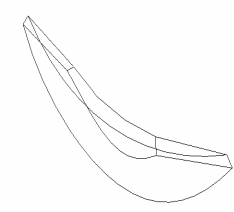

Figure2. The vane outline

\section{Structuring curve face of vane outline}

The curve face of vane outline is produced by the function of scanning using UG software. For the vane work face and the vane back face, the scanning leading line is the pattern curve formed by the control points of the cross points of upper and lower cross lines, and the scanning line is a straight line formed by the ends of upper and lower cross lines. Since there is the cast circular-angle at the cross line of the vane work face and the vane back face with the inter face and outer face of the flow road, and there is error between theory cross line and real cross line. To make the vane outline cross with the inter face and outer face of the flow road, we prolong the vane outline $3 \mathrm{~mm}$ along with the tangent line direction of the curve face. The redundant length needs to be cut by the inter face and outer face, and the curve face is sewed into real body, follow as Figure 3.

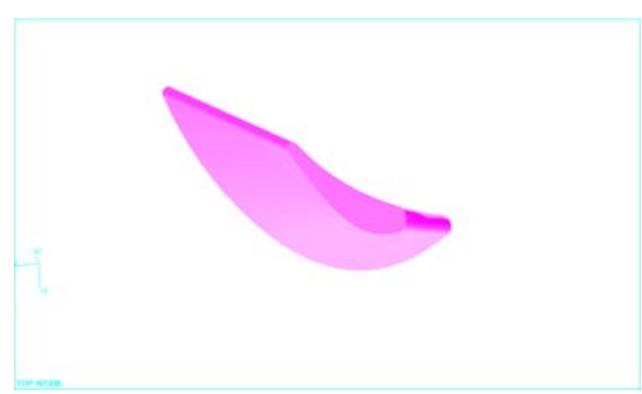

Figure3. The curve face of vane outline 


\section{Prolonging,Triming and Testing for the Curve Face of Vane Outline}

To test the error of real measure point with structuring curve face of the vane outline, using info/Distance information tool of single point with curve or curve face distance, the curve face will be colored from all screen show to large local area slowly, follow as Figure 4.

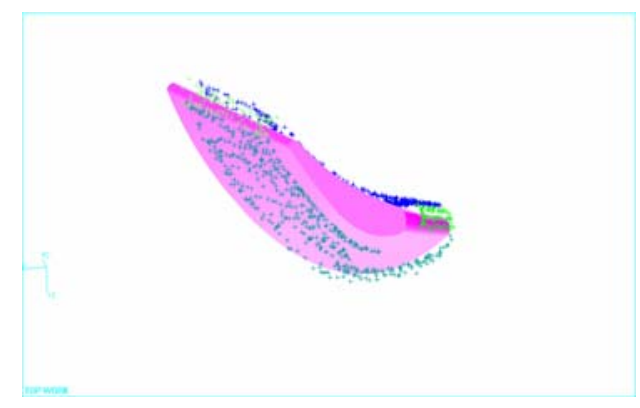

Figure4. The curve face of vane

In this time, since the data point always shows on the screen with “十”, which is not variable. After the data point is enlarged to certain degree, the big error point is blocked by curve face and disappears on the screen. If the errors of many points with curve face distance are bigger, we return to edit and to adjust the curve of the curve face modeling.

\section{3D MOdELING OF PILOT WHEEL}

The vane of pilot wheel is structured, its data are transfer into "Para solid", are exported to Solid edge software, and the surplus geometry feature is structured. This is usual geometry modeling, but the relations of measure point coordinate with surplus geometry feature must be in mind. 3D modeling follows as Figure 5.

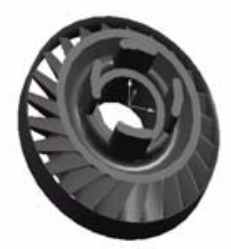

Figure5. The pilot wheel

\section{CONCLUSIONS}

The combination of reverse design and positive design provides a new design idea for production outer shape and structure design. Reverse design of production is the supplement and improving for positive CAD design and its function can not be replaced. In the design state of production concept, first the mud model or the timer model is made, then using 3D measure system the quick measure is made, and the modeling data is transferred into scattered and ordered 3D point cloud, finally using 3D CAD software 3D reconstruction is made and the ideal production is designed by positive design.

\section{REFERENCES}

[1] Ma Zhengyuan,Wang Huijun,Wang Dexi.Appling Principle of Reverse Engineering to remodel freeform surface of mould[J].Journal of Shenyang University of Technology,2004,26(4),pp.361-363

[2] Zhong Zhihua,Huang Haisha,Yang Xujing. Analysis of Data Selection and Optimization in Reverse Engineering[J].Journal of Hunan University(Natural Sciences),2004,31(4),pp.18-23

[3] Liu Hui. Research and Application on Reverse Engineering Used in NURBS Surface Modeling[J]. Journal of Engineering Graphics, 2009,30(2)

[4] Che Junhua,Li Shaozhen. The Smoothing Research of Complex Skew Curve Based on NURBS in Space[J]. Journal of Shandong University (Engineering Science),2002,32(4)

[5] Yu Dongjiu,Feng Yajuan. Research on reconstruction of product surfaces in reverse engineering[J].Machinery Design \& Manufacture, 2009,(7) 